\title{
Atrophic gastritis and enlarged gastric folds diagnosed by double-contrast upper gastrointestinal barium X-ray radiography are useful to predict future gastric cancer development based on the 3-year prospective observation
}

\author{
Nobutake Yamamichi $^{1}$ - Chigaya Hirano ${ }^{2}$ - Masao Ichinose ${ }^{3}$ Yu Takahashi $^{1}$. \\ Chihiro Minatsuki $^{1} \cdot$ Rie Matsuda $^{1} \cdot$ Chiemi Nakayama $^{1} \cdot$ Takeshi Shimamoto $^{1}$. \\ Shinya Kodashima ${ }^{1} \cdot$ Satoshi Ono $^{1} \cdot$ Yosuke Tsuji $^{1} \cdot$ Keiko Niimi $^{1}$ • \\ Yoshiki Sakaguchi $^{1}$ - Yosuke Kataoka ${ }^{1}$ Itaru Saito ${ }^{1}$ Itsuko Asada-Hirayama ${ }^{1}$. \\ Chihiro Takeuchi $^{1}$ - Seiichi Yakabi ${ }^{1}$ - Hikaru Kaikimoto ${ }^{1}$ - Yuta Matsumoto ${ }^{1}$. \\ Daisuke Yamaguchi ${ }^{1} \cdot$ Natsuko Kageyama-Yahara $^{1} \cdot$ Mitsuhiro Fujishiro $^{1}$. \\ Ryoichi Wada $^{2} \cdot$ Toru Mitsushima $^{2} \cdot$ Kazuhiko Koike $^{1}$
}

Received: 28 August 2015/Accepted: 6 October 2015/Published online: 20 October 2015

(C) The International Gastric Cancer Association and The Japanese Gastric Cancer Association 2015

\begin{abstract}
Background Double-contrast upper gastrointestinal barium X-ray radiography (UGI-XR) is the standard gastric cancer screening method in Japan. Atrophic gastritis and
\end{abstract}

enlarged gastric folds are considered the two major features of Helicobacter pylori-induced chronic gastritis, but the clinical meaning of evaluating them by UGI-XR has not been elucidated.
Nobutake Yamamichi

nyamamic-tky@umin.ac.jp

Chigaya Hirano

chigaya-kmc@umin.ac.jp

Masao Ichinose

ichinose@wakayama-med.ac.jp

Yu Takahashi

yutakahashi-tky@umin.ac.jp

Chihiro Minatsuki

furukawa-ymn@umin.ac.jp

Rie Matsuda

matsuda-ktz@umin.ac.jp

Chiemi Nakayama

nakayama-cib@umin.ac.jp

Takeshi Shimamoto

tsimamoto@gmail.com

Shinya Kodashima

kodashima-tky@umin.ac.jp

Satoshi Ono

satoshi-tky@umin.ac.jp

Yosuke Tsuji

ytsuji-tky@umin.ac.jp

Keiko Niimi

k-niimi@umin.ac.jp
Yoshiki Sakaguchi

sakaguchi-tky@umin.ac.jp

Yosuke Kataoka

saichu_resi_ky@yahoo.co.jp

Itaru Saito

itaru-s@el-saito.co.jp

Itsuko Asada-Hirayama

ihirayama-tky@umin.ac.jp

Chihiro Takeuchi

ctakeuchi-tky@umin.org

Seiichi Yakabi

syakabi@hotmail.com

Hikaru Kaikimoto

kakimoto-kh@umin.ac.jp

Yuta Matsumoto

matsumotoy-int@h.u-tokyo.ac.jp

Daisuke Yamaguchi

daisukehawks@gmail.com

Natsuko Kageyama-Yahara

natsukokageyama-tky@umin.ac.jp

Mitsuhiro Fujishiro

mtfujish-kkr@umin.ac.jp

Ryoichi Wada

suzy-w.makuhari@ruby.plala.or.jp 
Methods We analyzed healthy UGI-XR examinees without a history of gastrectomy, previous Helicobacter pylori eradication and usage of gastric acid suppressants. Results and conclusions Of the 6433 subjects, 1936 (30.1\%) had atrophic gastritis and 1253 (19.5\%) had enlarged gastric folds. During the 3-year prospective observational follow-up, gastric cancer developed in seven subjects, six of whom $(85.7 \%)$ had atrophic gastritis with H. pylori infection and five of whom $(71.4 \%)$ had enlarged gastric folds with $H$. pylori infection. The Kaplan-Meier method with log-rank testing revealed that both UGI-XRbased atrophic gastritis $(p=0.0011)$ and enlarged gastric folds $(p=0.0003)$ are significant predictors for future gastric cancer incidence.

Keywords Double-contrast upper gastrointestinal barium $\mathrm{X}$-ray radiography (UGI-XR) · Atrophic gastritis ·

Enlarged gastric folds - Gastric cancer · Helicobacter pylori

\section{Abbreviations \\ UGI-XR Double-contrast upper gastrointestinal barium $\mathrm{X}$-ray radiography \\ UGI-ES Upper gastrointestinal endoscopy \\ H. pylori Helicobacter pylori}

\section{Introduction}

Gastric cancer screening using double-contrast upper gastrointestinal barium X-ray radiography (UGI-XR) has been established and performed in Japan, where the mortality of gastric cancer is among the highest in the world [1, 2]. For more than 40 years, UGI-XR has been executed as the nationwide cancer screening program since there is substantial evidence of its efficacy in reducing mortality from gastric cancer [3-7]. Currently, however, UGI-XR is confronted with a variety of challenges, such as the low

Toru Mitsushima

mitsushima@kameda.jp

Kazuhiko Koike

kkoike-tky@umin.ac.jp

1 Department of Gastroenterology, Graduate School of Medicine, The University of Tokyo, 7-3-1, Hongo, Bunkyo-ku, Tokyo 113-8655, Japan

2 Kameda Medical Center Makuhari, CD-2, 1-3, Nakase, Mihama-ku, Chiba 261-0023, Japan

3 Second Department of Internal Medicine, Wakayama Medical University, 811-1, Kimiidera, Wakayama-shi, Wakayama 641-8509, Japan screening rate, non-negligible rate of adverse events particularly in the elderly, unfavorable influences of radiation exposure and a relatively low cancer detection rate compared with that from upper gastrointestinal endoscopy (UGI-ES) [8]. Given these challenges, gastric cancer screening by UGI-ES has been gradually increasing, especially for opportunistic screening [9]. In addition, various new screening methods using biological markers as the filter tests are now being investigated [10-16].

A fundamental difference exists between the conventional image-based methods and new serum marker-based screening methods: UGI-XR or UGI-ES is primarily aimed at the direct detection of gastric cancer. On the other hand, most biological marker-based methods have been developed to evaluate the pathological state of coexisting gastric mucosa and consequently assess the malignant potential in each subject $[13,14,16]$. At present, it is well-established that $H$. pylori carries a major risk of gastric cancer and also that $H$. pylori-associated chronic gastritis represents a precancerous condition of the stomach $[17,18]$. Therefore, effective screening will be possible if UGI-XR can provide additional information about the status of chronic gastritis other than cancer detection.

Atrophic gastritis (mucosal atrophy) and enlarged gastric folds (hypertrophic gastritis) have been reported as the two major features of $H$. pylori-associated gastritis diagnosed by UGI-XR [19-23]. Our recent study revealed that a high titer of serum anti-H. pylori $\operatorname{IgG}$ denoted by far the strongest association with "UGI-XR-based atrophic gastritis" among other significant factors such as current smoking, older age, male gender and so on [24]. Based on this background, the main aim of our present study is to clarify whether the evaluation of UGI-XR-based chronic gastritis can lead to prediction of gastric cancer development in the future.

\section{Methods}

\section{Study subjects}

The study population comprised 6901 asymptomatic general adults who underwent UGI-XR at Kameda Medical Center Makuhari (Chiba-shi, Chiba, Japan) in 2010, had no history of gastrectomy and also had sufficient data for analysis (Fig. 1a). Criteria for exclusion were the use of gastric acid suppressants or a history of $H$. pylori eradication therapy. This study was approved by the ethics committee of the University of Tokyo.

\section{Follow-up strategy}

We recommended that all the study subjects should undergo an annual health checkup including gastric cancer 
Fig. 1 Study flow chart (a) and typical images of chronic gastritis diagnosed by UGI-XR (b). The six images in $\mathbf{b}$ show a normal stomach with no gastritis (type $\mathrm{N}$ or $\mathrm{A}$, upper left), gastritis with modestly enlarged folds (type $\mathrm{M}$, upper middle), gastritis with gigantically enlarged folds (type $\mathrm{G}$, upper right), mild atrophic gastritis restrictively localized in the antrum and angle (type B, lower left), moderate atrophic gastritis extending from the antrum to the body and/or fornix (type $\mathrm{C}$, lower middle), and severe atrophic gastritis covering the entire stomach (type $\mathrm{D}$, lower right)

\section{a}

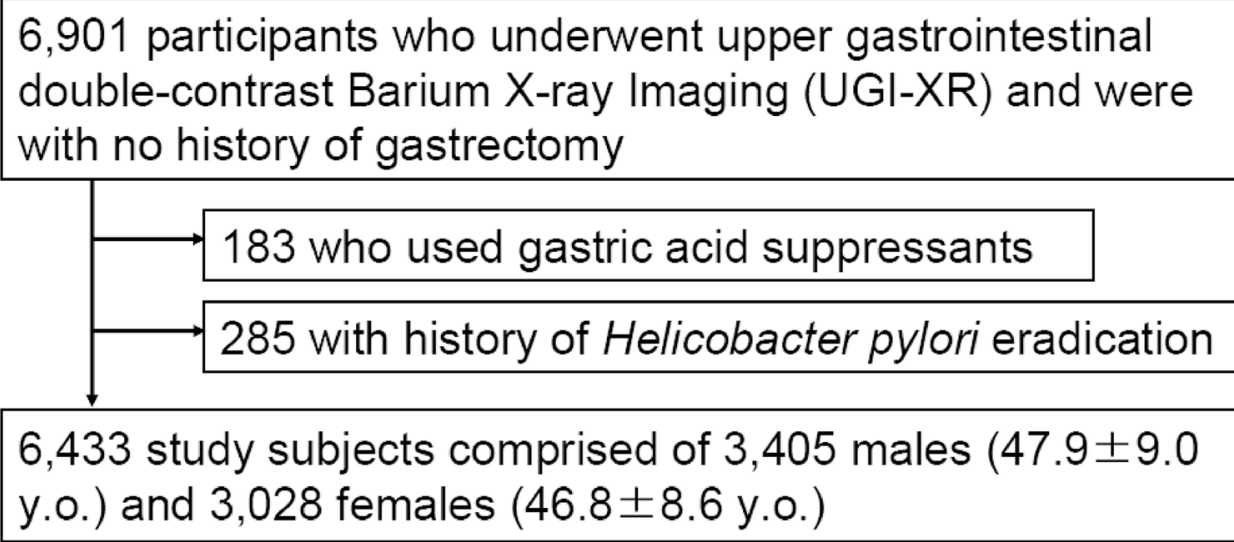

b

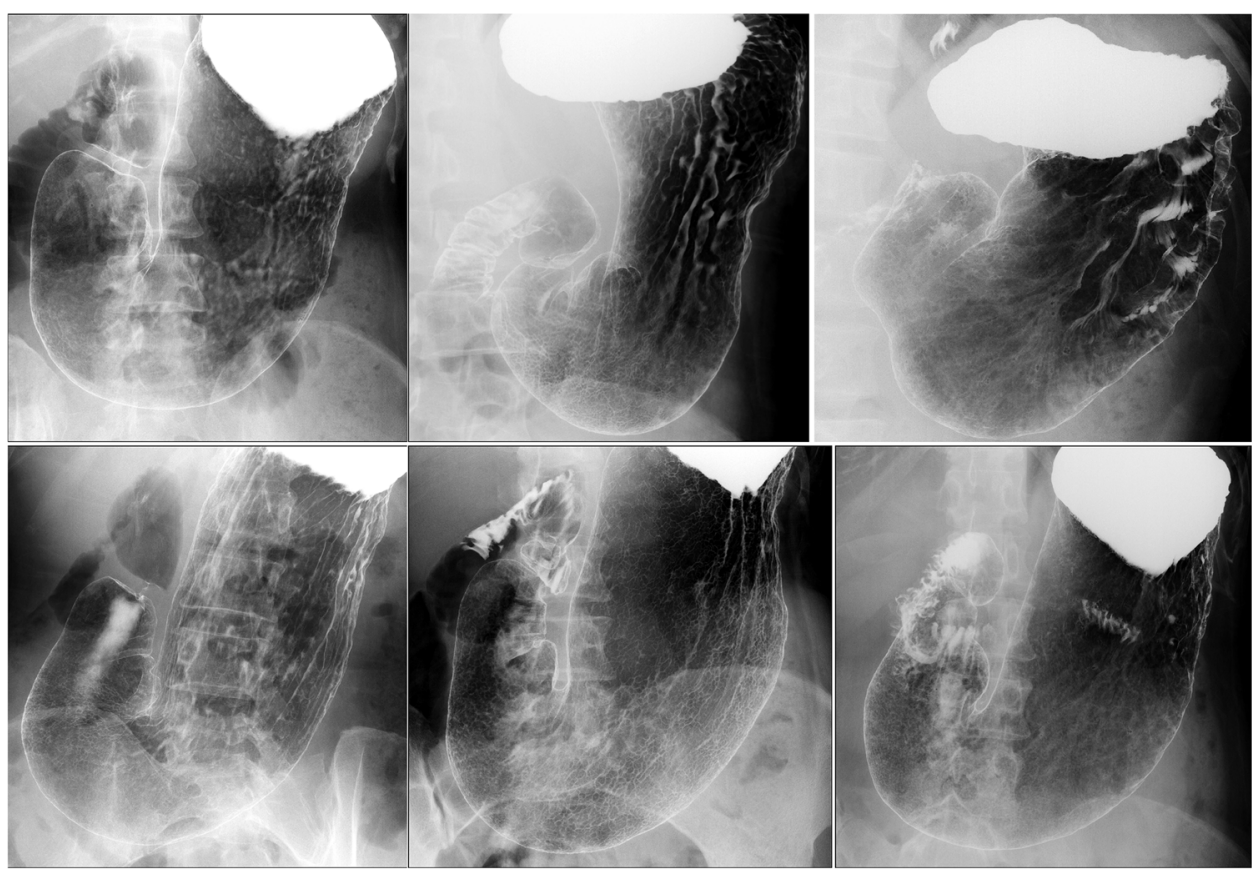

screening. All the data of UGI-XR and UGI-ES from all the study subjects were obtained from the beginning of 2010 to the end of 2013. Whether gastric cancer was detected by UGI-XR or UGI-ES, the final diagnosis was confirmed by histopathology using the biopsy specimens.

\section{Atrophic gastritis diagnosed by UGI-XR}

The method for UGI-XR has been previously described in detail [24]. In short, UGI-XR-based atrophic gastritis was classified into four types (Fig. 1b) as follows: no atrophic gastric mucosa (type A), no atrophic change observed on the entire gastric mucosa; mild atrophic gastritis (type B), slightly enlarged areae gastricae with some angularity and irregularity (mild mucosal atrophy) predominantly observed in the gastric antrum; moderate atrophic gastritis (type C), obviously enlarged areae gastricae with considerable angularity and irregularity (moderate mucosal atrophy) extending from the gastric antrum to body and/or fornix; severe atrophic gastritis (type D), very small or absent areae gastricae (severe mucosal atrophy) diffusely extending throughout the entire stomach.

\section{Enlarged gastric folds diagnosed by UGI-XR}

According to the previous reports [19-22], UGI-XR-based enlarged folds were classified into three types (Fig. 1b) as follows: normal gastric folds (type N), no enlarged folds with almost straight or marginally winding appearance observed in the gastric body; modestly enlarged gastric 
folds (type M), modestly enlarged folds in gently winding appearance observed in the gastric body; gigantically enlarged gastric folds (type $\mathrm{G}$ ): gigantically enlarged folds of tortuously winding appearance observed in the gastric body.

\section{Measurement of serum anti-Helicobacter pylori IgG and serum pepsinogens}

Serum anti-H. pylori IgG, pepsinogen I (PG I) and pepsinogen II (PG II) were measured using commercial kits (Eiken Chemical, Tokyo, Japan) as we previously reported [25-27].

\section{Statistical analyses}

Kaplan-Meier curves were generated to assess the incidence of gastric cancer using JMP 10 software (SAS Institute), and $p$ values $<0.05$ were considered statistically significant according to the log-rank test using the same software.

\section{Results}

\section{Characteristics of the study subjects}

From the 6901 UGI-XR examinees who agreed to participate in our study, we further excluded 183 users of gastric acid suppressants and 285 subjects with a history of $H$. pylori eradication (Fig. 1a). Consequently, we analyzed a total of 6433 subjects $(47.4 \pm 8.8$ years; range 20-83 years) comprising 3405 males and 3028 females. Of the total subjects, $1674(26.0 \%)$ tested positive for serum anti-H. pylori $\mathrm{IgG}$, which is almost equal to the recently reported prevalence of $H$. pylori infection in Japan [28].

\section{Atrophic gastritis surpasses enlarged gastric folds in diagnosing chronic $\boldsymbol{H}$. pylori infection by UGI-XR}

Of the 6433 series of UGI-XR images (Fig. 2a), 1936 $(30.1 \%)$ were diagnosed as atrophic gastritis (mild, $n=234$; moderate, $n=822$; severe, $n=880$ ), and 1253 $(19.5 \%)$ were diagnosed with enlarged gastric folds (modest, $n=968$; gigantic, $n=285$ ). In our study population (Fig. 2b), $95.9 \%(1202 / 1253)$ of subjects with enlarged gastric folds had atrophic gastritis. Conversely, only $62.1 \%(1202 / 1936)$ of subjects with atrophic gastritis had enlarged folds. A Venn diagram shows that the subjects with enlarged gastric folds were most likely to be individuals with atrophic gastritis (Fig. 2b). Our data suggested that atrophic gastritis has greater utility than enlarged gastric folds in evaluating $H$. pylori-induced chronic gastritis by UGI-XR.
"Atrophic gastritis" and "enlarged gastric folds" diagnosed by UGI-XR are both useful for predicting future development of gastric cancer

Until the end of the observation period, 4919 subjects underwent follow-up UGI-XR or UGI-ES at least once. Gastric cancer was detected in seven subjects $(0.14 \%)$, six of whom $(85.7 \%)$ had UGI-XR-based atrophic gastritis and were positive for serum anti-H. pylori IgG and five of whom $(71.4 \%)$ had UGI-XR-based enlarged folds and were also positive for serum anti-H. pylori IgG (Table 1).

Based on the 3-year follow-up data of the study subjects (mean follow-up period: $961 \pm 264$ days), Kaplan-Meier curves were plotted to assess the incidence of gastric cancer focusing on the two typical types of UGI-XR-based gastritis (Fig. 3). Log-rank testing showed that both UGIXR-based atrophic gastritis and UGI-XR-based enlarged folds are significant risk indicators for future gastric cancer development ( $p=0.0011$ and $p=0.0003$, respectively).

\section{Discussion}

This is the first large-scale study directly comparing "atrophic gastritis (mucosal atrophy)" and "enlarged gastric folds (hypertrophic gastritis)" diagnosed by UGI-XR. The inclusion relationship between them (Fig. 2b) suggests that "atrophic gastritis" is superior to "enlarged gastric folds" in detecting H. pylori-induced chronic gastritis. Nevertheless, we are of the opinion that the diagnosis of UGI-XR-based enlarged folds is also useful, because taking sufficient double-contrast barium $\mathrm{X}$-ray images for mucosal atrophy is usually more difficult than for enlarged folds, especially in case of inadequate barium coating or intragastric residual content. In anticipation of mutual compensation between atrophic gastritis and enlarged gastric folds, both types of UGI-XR-based chronic gastritis should be evaluated. Actually, prospective observation in our cohort showed that evaluation of both of these types of UGIXR-based chronic gastritis is useful for predicting future gastric cancer incidence (Fig. 3).

Despite the decreasing rate of $H$. pylori infection worldwide [29-32], the clinical significance of evaluating H. pylori-induced chronic gastritis is still quite high. Based on the present results, we recommend that UGI-XR should aim not only to detect gastric cancer itself, but also to evaluate atrophic gastritis and enlarged gastric folds, similar to the serum marker-based countermeasures aiming at the evaluation of the precancerous condition associated with chronic gastritis [14-16, 33]. We believe our present result will change the purpose of gastric cancer screening with barium X-ray radiography (UGI-XR) from "mere detection of gastric cancer" to "simultaneous evaluation of premalignant chronic gastritis." 
a

\begin{tabular}{|c|c|c|c|c|c|}
\hline & $\begin{array}{c}\text { No atrophic } \\
\text { gastric } \\
\text { mucosa }\end{array}$ & $\begin{array}{c}\text { Mild } \\
\text { atrophic } \\
\text { gastritis }\end{array}$ & $\begin{array}{c}\text { Moderate } \\
\text { atrophic } \\
\text { gastritis }\end{array}$ & $\begin{array}{c}\text { Severe } \\
\text { atrophic } \\
\text { gastritis }\end{array}$ & Total \\
\hline Normal gastric folds & $4,446(69.1 \%)$ & $189(2.9 \%)$ & $268(4.2 \%)$ & $277(4.3 \%)$ & $5,180(80.5 \%)$ \\
\hline Modestly enlarged folds & $51(0.8 \%)$ & $44(0.68 \%)$ & $466(7.2 \%)$ & $407(6.3 \%)$ & $968(15.0 \%)$ \\
\hline Gigantically enlarged folds & $0(0.0 \%)$ & $1(0.02 \%)$ & $88(1.4 \%)$ & $196(3.0 \%)$ & $285(4.4 \%)$ \\
\hline Total & $4,497(69.9 \%)$ & $234(3.6 \%)$ & $822(12.8 \%)$ & $880(13.7 \%)$ & $6,433(100 \%)$ \\
\hline
\end{tabular}

b

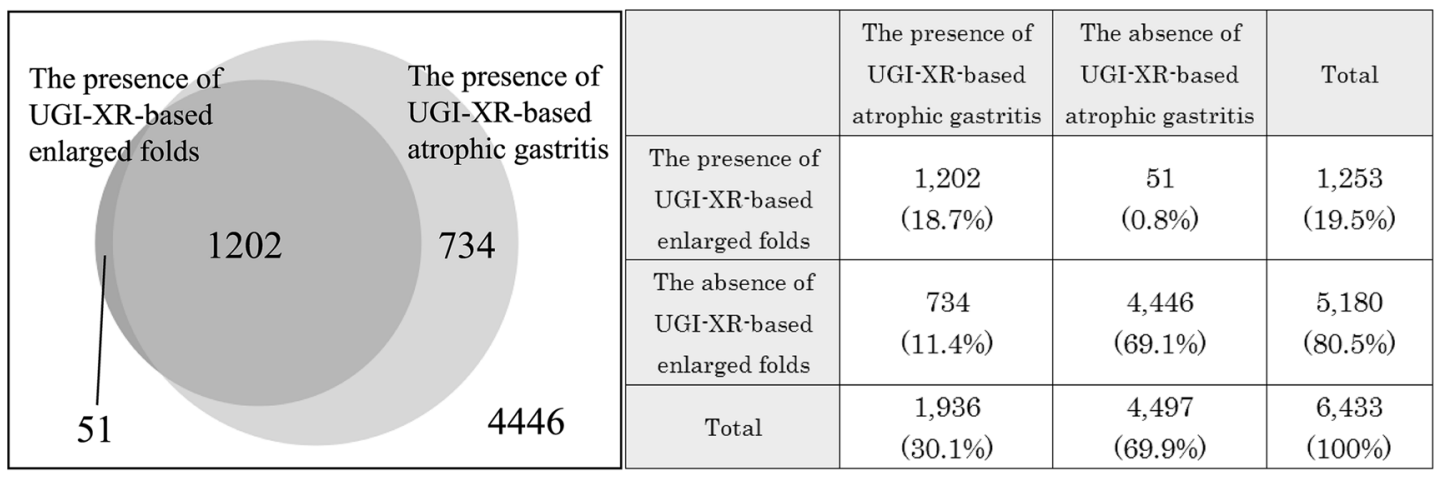

Fig. 2 Relationship between atrophic gastritis (normal, mild, moderate, severe) and enlarged gastric folds (normal, modest, gigantic) diagnosed by double-contrast upper gastrointestinal barium X-ray radiography (UGI-XR). a A $3 \times 4$ contingency table showing the distribution of atrophic gastritis and enlarged gastric folds. b A Venn diagram and $2 \times 2$ contingency table showing the relationship between atrophic gastritis and enlarged gastric folds

Table 1 Characteristics of the seven subjects who developed gastric cancer in the 3-year follow-up

\begin{tabular}{lllllrrrrr}
\hline Follow-up years & Age & Sex & $\begin{array}{l}\text { UGI-XR-based atrophic } \\
\text { gastritis (normal, mild, } \\
\text { moderate, severe) }\end{array}$ & $\begin{array}{l}\text { UGI-XR-based enlarged } \\
\text { gastric folds (normal, } \\
\text { modest, gigantic) }\end{array}$ & $\begin{array}{l}\text { Anti- } H P \\
\text { IgG }( \pm)\end{array}$ & $\begin{array}{l}\text { Anti- } H P \text { IgG } \\
(\mathrm{U} / \mathrm{ml})\end{array}$ & $\begin{array}{l}\text { PG I } \\
(\mathrm{ng} / \mathrm{ml})\end{array}$ & $\begin{array}{l}\text { PG II } \\
(\mathrm{ng} / \mathrm{ml})\end{array}$ & $\begin{array}{l}\text { PG I/II } \\
\text { ratio }\end{array}$ \\
\hline 0 & 72 & $\mathrm{M}$ & + (severe) & + (modest) & + & 112.5 & 77.9 & 32.5 & 2.4 \\
1 & 50 & $\mathrm{~F}$ & + (severe) & + (modest) & + & 59.8 & 79.2 & 30.4 & 2.6 \\
2 & 59 & $\mathrm{M}$ & +(moderate) & - (normal) & + & 16.0 & 41.5 & 9.6 & 4.3 \\
3 & 58 & $\mathrm{M}$ & +(severe) & + (modest) & + & 32.2 & 57.2 & 25.7 & 2.2 \\
3 & 54 & $\mathrm{M}$ & + (severe) & +(modest) & + & 26.6 & 33.9 & 20.4 & 1.7 \\
3 & 55 & $\mathrm{M}$ & -(normal) & -(normal) & - & 1.1 & 78.4 & 15.8 & 5.0 \\
3 & 64 & $\mathrm{M}$ & +(severe) & +(modest) & + & 19.3 & 27.1 & 18.6 & 1.5 \\
\hline
\end{tabular}

$U G I-X R$ double-contrast upper gastrointestinal barium X-ray radiography, $P G$ pepsinogen, $H P$ Helicobacter pylori, $M$ males, $F$ females

Fig. 3 Kaplan-Meier curves to assess the 3-year incidences of gastric cancer focusing on the UGI-XR-based atrophic gastritis (a) and UGI-XR-based enlarged gastric folds (b). All the censored subjects are denoted by the tick marks. A $p$ value less than 0.05 was considered statistically significant according to the log-rank test a

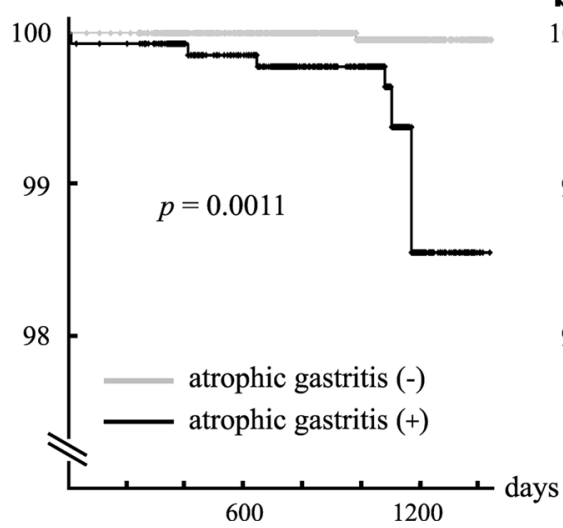

b

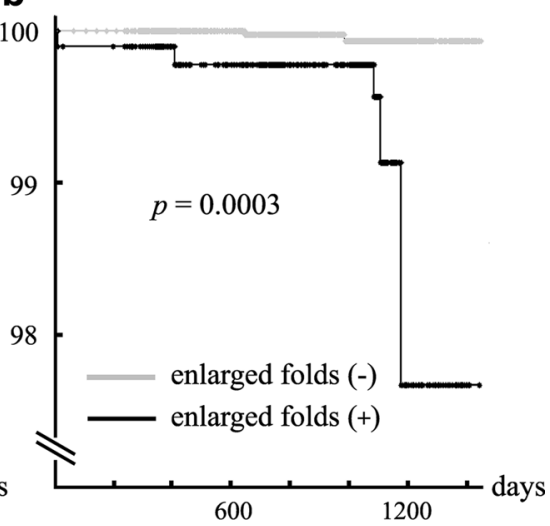




\section{Conclusions}

Prospective observation of 6433 generally healthy subjects for 3 years showed that atrophic gastritis and enlarged gastric folds diagnosed by double-contrast upper gastrointestinal barium X-ray radiography (UGI-XR) are both useful for predicting future gastric cancer incidence.

Acknowledgments This work was supported by a Grant-in-Aid for Scientific Research (C) from the Japan Society for the Promotion of Science (grant no. 25460381).

\section{Compliance with ethical standards}

Conflict of interest All authors declare no conflict of interest.

Human rights statement and informed consent All procedures followed were in accordance with the ethical standards of the responsible committee on human experimentation (institutional and national) and with the Helsinki Declaration of 1964 and later versions. Informed consent was obtained from all patients for being included in the study.

\section{References}

1. Leung WK, Wu MS, Kakugawa Y, Kim JJ, Yeoh KG, et al. Screening for gastric cancer in Asia: current evidence and practice. Lancet Oncol. 2008;9:279-87.

2. Crew KD, Neugut AI. Epidemiology of gastric cancer. World J Gastroenterol. 2006;12:354-62.

3. Lee KJ, Inoue M, Otani T, Iwasaki M, Sasazuki S, et al. Gastric cancer screening and subsequent risk of gastric cancer: a largescale population-based cohort study, with a 13-year follow-up in Japan. Int J Cancer. 2006;118:2315-21.

4. Oshima A, Hirata N, Ubukata T, Umeda K, Fujimoto I. Evaluation of a mass screening program for stomach cancer with a case-control study design. Int J Cancer. 1986;38:829-33.

5. Hisamichi S, Sugawara N. Mass screening for gastric cancer by X-ray examination. Jpn J Clin Oncol. 1984;14:211-23.

6. Mizoue T, Yoshimura T, Tokui N, Hoshiyama Y, Yatsuya H, et al. Prospective study of screening for stomach cancer in Japan. Int J Cancer. 2003;106:103-7.

7. Tsubono Y, Hisamichi S. Screening for gastric cancer in Japan. Gastric Cancer. 2000;3:9-18.

8. Ohata H, Oka M, Yanaoka K, Shimizu Y, Mukoubayashi C, et al. Gastric cancer screening of a high-risk population in Japan using serum pepsinogen and barium digital radiography. Cancer Sci. 2005;96:713-20.

9. Hosokawa O, Miyanaga T, Kaizaki Y, Hattori M, Dohden K, et al. Decreased death from gastric cancer by endoscopic screening: association with a population-based cancer registry. Scand J Gastroenterol. 2008;43:1112-5.

10. Ichinose M, Yahagi N, Oka M, Ikeda H, Omata M. Screening for Gastric Cancer in Japan. In: Wu GY, Aziz K, editors. Cancer screening: practical guide for physicians. Humana Press Publisher; 2001. p. 255-68.

11. Maekita T, Nakazawa K, Mihara M, Nakajima T, Yanaoka K, et al. High levels of aberrant DNA methylation in Helicobacter pylori-infected gastric mucosae and its possible association with gastric cancer risk. Clin Cancer Res. 2006;12:989-95.

12. Ando T, Yoshida T, Enomoto S, Asada K, Tatematsu M, et al. DNA methylation of microRNA genes in gastric mucosae of gastric cancer patients: its possible involvement in the formation of epigenetic field defect. Int J Cancer. 2009;124:2367-74.

13. Aikou S, Ohmoto Y, Gunji T, Matsuhashi N, Ohtsu H, et al. Tests for serum levels of trefoil factor family proteins can improve gastric cancer screening. Gastroenterology. 2011;141:837-45.

14. Ohata H, Kitauchi S, Yoshimura N, Mugitani K, Iwane M, et al. Progression of chronic atrophic gastritis associated with Helicobacter pylori infection increases risk of gastric cancer. Int $\mathbf{J}$ Cancer. 2004;109:138-43.

15. Watabe H, Mitsushima T, Yamaji Y, Okamoto M, Wada R, et al. Predicting the development of gastric cancer from combining Helicobacter pylori antibodies and serum pepsinogen status: a prospective endoscopic cohort study. Gut. 2005;54:764-8.

16. Mukoubayashi C, Yanaoka K, Ohata H, Arii K, Tamai H, et al. Serum pepsinogen and gastric cancer screening. Intern Med. 2007;46:261-6.

17. Polk DB, Peek RM Jr. Helicobacter pylori: gastric cancer and beyond. Nat Rev Cancer. 2010;10:403-14.

18. Correa P, Houghton J. Carcinogenesis of Helicobacter pylori. Gastroenterology. 2007;133:659-72.

19. Sohn J, Levine MS, Furth EE, Laufer I, Rubesin SE, et al. Helicobacter pylori gastritis: radiographic findings. Radiology. 1995;195:763-7.

20. Yamamichi N, Shimamoto T, Minatsuki C, Yoshida Y, Fujishiro $\mathrm{M}$, et al. Postprandial fullness correlates with rapid inflow of gastric content into duodenum but not with chronic gastritis. BMC Gastroenterol. 2011;11:140.

21. Rubesin SE, Levine MS, Laufer I. Double-contrast upper gastrointestinal radiography: a pattern approach for diseases of the stomach. Radiology. 2008;246:33-48.

22. Dheer S, Levine MS, Redfern RO, Metz DC, Rubesin SE, et al. Radiographically diagnosed antral gastritis: findings in patients with and without Helicobacter pylori infection. Br J Radiol. 2002;75:805-11.

23. Yamamichi N, Hirano C, Takahashi Y, Minatsuki C, Nakayama $\mathrm{C}$, et al. Comparative analysis of upper gastrointestinal endoscopy, double-contrast upper gastrointestinal barium X-ray radiography, and the titer of serum anti-Helicobacter pylori $\mathrm{IgG}$ focusing on the diagnosis of atrophic gastritis. Gastric Cancer. 2015. [Epub ahead of print].

24. Yamamichi N, Hirano C, Shimamoto T, Minatsuki C, Takahashi $\mathrm{Y}$, et al. Associated Factors of atrophic gastritis diagnosed by double-contrast upper gastrointestinal barium x-ray radiography: a cross-sectional study analyzing 6901 healthy subjects in Japan. PLoS One. 2014;9:e111359.

25. Minatsuki C, Yamamichi N, Shimamoto T, Kakimoto H, Takahashi $\mathrm{Y}$, et al. Background factors of reflux esophagitis and nonerosive reflux disease: a cross-sectional study of 10,837 subjects in Japan. PLoS One. 2013;8:e69891.

26. Shimamoto $\mathrm{T}$, Yamamichi $\mathrm{N}$, Kodashima $\mathrm{S}$, Takahashi $\mathrm{Y}$, Fujishiro M, et al. No association of coffee consumption with gastric ulcer, duodenal ulcer, reflux esophagitis, and non-erosive reflux disease: a cross-sectional study of 8013 healthy subjects in Japan. PLoS One. 2013;8:e65996.

27. Takahashi $\mathrm{Y}$, Yamamichi $\mathrm{N}$, Shimamoto $\mathrm{T}$, Mochizuki $\mathrm{S}$, Fujishiro M, et al. Helicobacter pylori infection is positively associated with gallstones: a large-scale cross-sectional study in Japan. J Gastroenterol. 2014;49:882-9.

28. Hirayama Y, Kawai T, Otaki J, Kawakami K, Harada Y. Prevalence of Helicobacter pylori infection with healthy subjects in Japan. J Gastroenterol Hepatol. 2014;29(Suppl 4):16-9.

29. Nakajima S, Nishiyama Y, Yamaoka M, Yasuoka T, Cho E. Changes in the prevalence of Helicobacter pylori infection and gastrointestinal diseases in the past 17 years. J Gastroenterol Hepatol. 2010;25(Suppl 1):S99-110. 
30. Lee SY, Park HS, Yu SK, Sung IK, Jin CJ, et al. Decreasing prevalence of Helicobacter pylori infection: a 9-year observational study. Hepatogastroenterology. 2007;54:630-3.

31. Brown LM. Helicobacter pylori: epidemiology and routes of transmission. Epidemiol Rev. 2000;22:283-97.

32. Bures J, Kopacova M, Koupil I, Seifert B, Skodova Fendrichova M, et al. Significant decrease in prevalence of Helicobacter pylori in the Czech Republic. World J Gastroenterol. 2012;18:4412-8.
33. Yoshida T, Kato J, Inoue I, Yoshimura N, Deguchi H, et al. Cancer development based on chronic active gastritis and resulting gastric atrophy as assessed by serum levels of pepsinogen and Helicobacter pylori antibody titer. Int $\mathrm{J}$ Cancer. 2014;134:1445-57. 